\title{
Behavior of volatiles during the Giant Impact
}

\author{
RENATA B. SCHAAN ${ }^{1,2}$ AND RAZVAN CARACAS ${ }^{2,3}$ \\ ${ }^{1}$ Ecole Normale Superieure de Lyon \\ ${ }^{2}$ CNRS \\ ${ }^{3}$ University of Oslo \\ Presenting Author: renatabrandelli@gmail.com
}

Silica is a major rock forming material in our Solar System. The formation of rocky bodies depends on accretion and giant impacts, which often produce silicate fluids. The most recent theory of Giant Impact for the Moon formation implicates in the formation of a synesthia in the aftermath of the impact. In this scenario, a hot silicate atmosphere would have formed and if there would be an ocean, it would be vaporized as well together with the material. Although the proto Earth and the impactor most likely had different compositions before they collide, the Moon and the Earth end up sharing similar isotopic composition, with the exception of the volatiles elements. These relations are yet to be explained and can be study by the liquid-vapor relation in single and complex systems.

Placing the critical point of the $\mathrm{SiO}_{2}-\mathrm{H}_{2} \mathrm{O}$ binary system affects melting temperatures, solubility of minerals and concentration of mobile elements, which has implications for understanding the conditions at the formation of the Earth-Moon system, but also for other geological scenarios such as subducction zones.

We do first-principles molecular dynamics modeling of the $\mathrm{SiO}_{2}-\mathrm{H}_{2} \mathrm{O}$ binary system during the Giant Impact employing the Vienna Ab Initio Simulation Package (VASP) implementation of the Projector Augmented Wavefunction (PAW) method. We use cristobalite with $72 \mathrm{SiO}_{2}$ units as a starting condition. The material was heated up to $2000 \mathrm{~K}$ and $4000 \mathrm{~K}$ and then 8 and 72 units of $\mathrm{H}_{2} \mathrm{O}$ were added to the system. We present initial results.

Acknowledgements: This research is supported by the European Research Council (ERC) under the European Union's Horizon 2020 research and innovation programme (grant agreement \#681818 IMPACT). The simulations were performed on the GENCI supercomputers (eDARI/CINES grants x106368). 